\title{
Clinical, Histologic, and Immunophenotypic Features of Serrated Polyps in Patients With Inflammatory Bowel Disease
}

\author{
Chen Yang ${ }^{\mathrm{a}}$, Yaman Tarabishy ${ }^{\mathrm{b}}$, Themistocles Dassopoulos ${ }^{\mathrm{c}}$, \\ ILKe Nalbantoglu ${ }^{\text {a,d }}$
}

\begin{abstract}
Background: Colorectal serrated polyps (SP), which include hyperplastic polyps (HP), sessile serrated adenomas/polyps (SSA/P), and traditional serrated adenomas, are not uncommon and have been implicated to play a role in the pathogenesis in a subset of sporadic colorectal carcinomas; however, their significance in patients with prolonged inflammatory bowel disease (IBD) remains unclear.
\end{abstract}

Methods: We retrospectively studied the clinicopathologic features, BRAF and $\beta$-catenin immunohistochemistry staining patterns in 36 SPs from 28 patients with IBD compared with 40 SPs in patients without IBD.

Results: Eleven SSA/Ps and $25 \mathrm{HPs}$ from IBD and site-matched controls were included. SSA/Ps in the study group were slightly more commonly seen in males $(55 \%$ vs. $41 \%, \mathrm{P}=0.7)$ and older patients (55.2 vs. 47.8 years, $\mathrm{P}=0.2)$ compared to patients with HP. They were moderately larger $(7.13 \mathrm{~mm}$ vs. $4.83 \mathrm{~mm}, \mathrm{P}=0.14)$ and more likely located on the right $(63.6 \%$ vs. $32 \%, \mathrm{P}=0.46)$. Smaller percentage of SSA/Ps showed BRAF staining compared to controls $(55.6 \%$ vs. $73.3 \%, \mathrm{P}=0.41)$ and HPs showed similar features $(52.0 \%$ vs. $54.2 \%, \mathrm{P}=1) . \beta$-catenin was negative in all cases. During follow-up, only one patient in the SSA/P group developed carcinoma 42 months after at the same site and two developed adenoma-like low-grade dysplasia but no patients with HPs had such findings.

Conclusions: Our findings show that SPs in IBD share similar clinicodemographic and immunophenotypical features with sporadic SPs. However, patients with SSA/Ps may have a slight increase in risk of developing dysplasia compared to patients with HPs in IBD.

Manuscript submitted July 17, 2018, accepted July 30, 2018

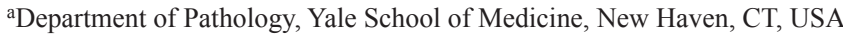
bPeoria Tazewell Pathology Group, Peoria, IL, USA

'Baylor University Medical Center, Baylor Scott and White Center for Inflammatory Bowel Diseases, Dallas, TX, USA

${ }^{\mathrm{d} C o r r e s p o n d i n g ~ A u t h o r: ~ I L K e ~ N a l b a n t o g l u, ~ D e p a r t m e n t ~ o f ~ P a t h o l o g y / S u r g i c a l ~}$ Pathology-EP2-608B, 310 Cedar Street, New Haven, CT 06515, USA. Email: ilke.nalbantoglu@yale.edu

doi: https://doi.org/10.14740/gr1064w
Keywords: Serrated; Polyps; Inflammatory bowel disease; BRAF; $\beta$-catenin

\section{Introduction}

Patients with inflammatory bowel disease (IBD), in the form of chronic ulcerative colitis (UC) or Crohn's disease, have a well-recognized increased risk of developing colorectal cancer that increases with the duration of inflammatory activity [1]. Other risk factors for the development of colorectal cancer in these patients include the extent and intensity of inflammation [2-4]. Expert consensus recommends endoscopic surveillance with random colonic mucosal biopsies for patients with long-standing ( 8 years or greater) IBD that involves more than one-third of the entire colon [5-8]. The goal of this approach is to detect dysplasia, further categorized as either low-grade or high-grade, which is currently the most useful and reproducible risk marker of malignancy [9]. Current consensus suggests endoscopic removal of polypoid and non-polypoid endoscopically visible and resectable dysplasia, while patients with endoscopically invisible dysplasia should be referred to and evaluated by an endoscopist with expertise in using chromoendoscopy with high-definition colonoscopy $[7,8,10]$.

Colorectal serrated polyps (SP), which include hyperplastic polyps (HP), sessile serrated adenomas/polyps (SSA/P), and traditional serrated adenomas (TSA), are not uncommon and have been implicated to play a role in the pathogenesis in a subset of sporadic colorectal carcinomas [11, 12]. SPs in general population have been widely studied and have been associated with the pathogenesis of at least $20 \%$ of colorectal carcinomas $[11,12]$. However, the classification and biological behavior of SPs in the setting of IBD still remains a continuous debate. The literature on this topic is limited with most papers published to date reporting that SPs in IBD patients behave similar to sporadic ones [13-15].

$B R A F$ mutation has been known to be a key factor in the serrated pathway progression of colorectal cancers and has recently been detected in SPs in both the IBD and sporadic setting $[16,17]$. However, the status of the BRAF mutation has not been extensively studied on SPs, especially those in the background of IBD. However, a rare study reports simi- 
lar findings to sporadic SSA/Ps [13]. $\beta$-catenin is the marker that can be detected in the nucleus of cells in the conventional adenoma that are associated with the activation of WNT signaling pathway and carcinogenesis. A recent study has shown that $\beta$-catenin nuclear staining is less common in sporadic SPs compared to conventional adenomas [18]. However, the utility of $\beta$-catenin in SPs associated with IBD have not yet been studied.

Nevertheless, recognition of the full range of histologic lesions with malignant potential in the setting of IBD is incomplete. To our knowledge, systematic investigations comparing the histopathologic and immunophenotypic features of SPs in patients with IBD and normal subjects are limited. Therefore, we undertook a retrospective approach to evaluate SPs diagnosed within our department over a 10 -year period of time.

\section{Materials and Methods}

\section{Case selection}

This study was approved by the Human Research Protection Office of Washington University in St. Louis. A retrospective search of the pathology files of Washington University/BarnesJewish Hospital and was conducted between 2004 and 2014. Patients with biopsy or resection specimens with a diagnosis of "hyperplastic polyp", "hyperplastic change", "sessile", or "serrated" and a history of IBD were selected. Basic demographics data including age and sex, duration and type of IBD, endoscopic findings, total number of colonoscopies during follow-up, extent of disease, polyp site and size, background mucosa, concurrent dysplasia/cancer and follow-up information were recorded. Control cases consisted of 40 consecutive, well-oriented, site matched HPs and SSA/Ps in non-IBD patients who underwent colonoscopy for routine cancer surveillance. Cases with rectal polyps, poorly oriented specimens, and/or unavailability of archived materials were excluded. A total of $11 \mathrm{SSA} / \mathrm{Ps}$ and $25 \mathrm{HPs}$ in the IBD group and $15 \mathrm{SSA}$ Ps and 25 HPs in the control group were included in this study.

\section{Histologic and immunohistochemical (IHC) analysis}

The cases were classified as either SSA/P or HP through blinded histological review by two pathologists (Y. T. and I. N.) based on the WHO criteria [19]. The disagreements were resolved by consensus review.

Immunohistochemistry was performed on $4 \mu \mathrm{m}$ sections from formalin-fixed, paraffin-embedded tissue blocks on a Ventana Benchmark XT automated immunostainer (Ventana Medical Systems, Inc., Tucson, AZ) using standard established laboratory protocols with monoclonal antibody to $\beta$-catenin (Ventana; clone 14; monoclonal; prediluted). Ventana's ultraView Universal DAB Detection Kit was utilized as the secondary antibody cocktail to detect binding of the primary antibody which was subsequently visualized using hydrogen peroxide substrate and a 3, 3'-diaminobenzidine tetrahydrochloride (DAB) chromogen. Manufacturer antigen retrieval condi- tions were used (Ventana CC1, EDTA-Tris, $\mathrm{pH} 8.0$ solution). Commercially available monoclonal antibodies for BRAF (NewEast Biosciences, clone V600e; monoclonal; 1:100 dilution) was utilized. The slides were manually stained. Antigen retrieval was by heat mediation in citrate buffer ( $\mathrm{pH} 6.0$ solution). Samples were then incubated with primary antibody (1:100) overnight at $4{ }^{\circ} \mathrm{C}$. A horseradish peroxidase-conjugated goat anti-mouse IgG (dilution 1:50) was used as secondary antibody. Proper positive and negative controls were utilized (colonic adenocarcinoma with confirmed $B R A F$ V600e mutation by molecular analysis for $B R A F$, tubular adenoma for $\beta$-catenin).

Immunoreactivity was evaluated in blinded fashion by at least two of the three pathologists (C.Y., Y. T. or I. N.). For BRAF stain, cytoplasmic staining was considered positive, regardless of its intensity. Complete absence of cytoplasmic positivity was considered negative. Membranous $\beta$-catenin was considered normal, whereas loss of cytoplasmic positivity with nuclear reactivity was considered positive (mutated).

\section{Statistical analysis}

The Fisher exact test was used to compare percentage of cases. The Wilcoxon-Mann-Whitney test was used to compare means. P value less than 0.05 was considered statistically significant.

\section{Results}

\section{General patient characteristics and endoscopic features}

A total of 36 lesions from 28 IBD patients were identified. The mean age was 50.7 years (range: 29 to 75 years). There was no significant gender predilection, as 13 cases were male (46\%) and 15 cases were female (54\%). Sixteen patients were diagnosed of UC (57\%) and the remainder 12 had Crohn's disease $(43 \%)$. None of the study subjects had indeterminate colitis. The overall disease duration was 15.8 years (range: 1 to 40 years).

The majority the lesions were polypoid in appearance endoscopically $(32,89 \%)$, whereas the remainder $4(11 \%)$ were described as flat lesions with two described as granular mucosal changes and the other two described as mild erythematous mucosa. Three of these flat lesions were HPs and one was SSA/P. Seventeen polyps (47\%) were noted in the right and 19 $(53 \%)$ on the left. The mean polyp size was $5.74 \mathrm{~mm}$ (range: 2 to $15 \mathrm{~mm}$ ) with SSA/Ps measuring $7.13 \mathrm{~mm}$ (range: 3 to 12 $\mathrm{mm}$ ) and HPs measuring $4.83 \mathrm{~mm}$ (range: 1 to $15 \mathrm{~mm}$ ).

\section{Clinicopathologic and immunohistochemical features}

The study group consisted of 36 polyps from 28 patients, which were reviewed and classified by two pathologists (Y. T. and I. N.) to either SSA/P $(n=11)$ or HP $(n=25)$ using the WHO criteria [19]. The general clinical, histologic and IHC character- 
Table 1. Characteristics of Inflammatory Bowel Disease Patients With Serrated Lesions (2004 to 2014)

\begin{tabular}{llll}
\hline Feature & HP & SSA/P & P value \\
\hline Number of cases (number of patients) & $25(17$ pts $)$ & 11 (11 pts) & 0.2 \\
Age (y), mean (range) & $47.8(29$ to 75$)$ & $55.2(29$ to 68$)$ & 0.7 \\
Male/female & $7 / 10$ & $6 / 5$ & 0.25 \\
IBD type (UC vs. CD) & $14: 11$ & $8: 3$ & 0.42 \\
Mean IBD duration at index, years (IQR) & $17.7(1$ to 40$)$ & $12.7(3$ to 20$)$ & 1 \\
Polyp/flat lesion & $22 / 3$ & $10 / 1$ & 0.46 \\
Right/left colon & $9 / 11$ & $7 / 4$ & \\
\hline
\end{tabular}

Pts: patients; y: years.

istics of these lesions are summarized in Tables 1 and 2.

A total of $11(31 \%) \mathrm{SSA} / \mathrm{Ps}$ in the study group were identified. Six of the patients were male (55\%) and five of the patients were female $(45 \%)$. The mean patient age was 55.2 (range: 29 to 68 years). Eight cases (73\%) were from patients with UC, while three were patients with Crohn's disease (27\%). The mean duration of IBD for SSA/P was 12.7 years (range: 3 to 20 years). Of the SSA/Ps, seven (64\%) were located in the right colon with size of $7.13 \mathrm{~mm}$ (range: 3 to $12 \mathrm{~mm}$ ). Ninety percent of them (10 cases) were defined as polyp on endoscopy, whereas only one lesion was defined as flat. The mean polyp size was $7.13 \mathrm{~mm}$. The background mucosa showed chronic active colitis at the surrounding mucosa in three cases, and was normal in the remainder. BRAF staining (available in nine cases in study group) was positive in $55.6 \%$ of the SSA/Ps for the study group, compared to $73.3 \%$ of the control group.

A total of $25(70 \%)$ HPs were identified in the study group. Seven $(41 \%)$ of the cases were male, whereas $10(59 \%)$ of the cases were female. The mean patient age was 47.8 (range: 29 to 75 years). Fourteen cases $(56 \%)$ were from patients with ulcerative colitis, while 11 (44\%) had Crohn's disease. The mean duration of IBD was 17.7 years (range: 1 to 40 years). Nine lesions (36\%) were located in the right colon and 11 lesions $(44 \%)$ in the left colon. Location data were not available for five $(20 \%)$ of the HPs. Eighty-eight percent of them (22 cases) were defined as "polypoid" on endoscopy, whereas three lesions were defined as either granular mucosal changes or mildly erythematous mucosa. The mean polyp size was 4.83 $\mathrm{mm}$ (range: 1 to $15 \mathrm{~mm}$ ). BRAF staining (available in 25 cases in the study group) was positive in $52 \%$ of the HPs for the study group (13/25), which was similar to the control group $(54.2 \%, 13 / 24)$. The background mucosa showed chronic active colitis at the surrounding mucosa in three cases, and no histopathologic abnormalities in the remainder.

$\mathrm{SSA} / \mathrm{Ps}$ in IBD were slightly more common in males $(55 \%$ vs. $41 \%, \mathrm{P}=0.7)$, older patients (55.2 vs. 47.8 years, $\mathrm{P}=0.2)$, in the right colon $(63.6 \%$ vs. $32 \%, \mathrm{P}=0.46)$, and were larger in size ( 7.13 vs. $4.83 \mathrm{~mm}, \mathrm{P}=0.14)$ compared to HPs in IBD. However, IBD patients with SSA/Ps had shorter disease duration (12.7 vs. 17.7 years, $\mathrm{P}=0.42$ ) compared to IBD patients with HPs.

Overall, a total of $18(53 \%)$ cases in the study group were

Table 2. Staining Pattern for Ki-67 and BRAF for HP and SSA/P

\begin{tabular}{|c|c|c|c|c|}
\hline & \multicolumn{2}{|c|}{ Study group } & \multicolumn{2}{|c|}{ Control group } \\
\hline & SSA/P & HP & SSA/P & HP \\
\hline \multicolumn{5}{|l|}{ Site } \\
\hline Right (\%) & $7(63.6 \%)$ & $10(40.0 \%)$ & $11(73.3 \%)$ & $10(40.0 \%)$ \\
\hline $\mathrm{P}$ value & 0.68 & 1 & & \\
\hline Positive (\%) & $5(55.6 \%)$ & $13(52.0 \%)$ & $11(73.3 \%)$ & $13(54.2 \%)$ \\
\hline Negative (\%) & $4(44.4 \%)$ & $12(48.0 \%)$ & $4(26.7 \%)$ & $11(45.8 \%)$ \\
\hline $\mathrm{P}$ value & 0.41 & 1 & & \\
\hline \multicolumn{5}{|l|}{$\beta$-catenin } \\
\hline Positive (\%) & $0(0 \%)$ & $0(0 \%)$ & $0(0 \%)$ & $0(0 \%)$ \\
\hline
\end{tabular}

*Two cases of SSA/P in study group and one HP from the control group were excluded from BRAF evaluation with inadequate tissue for interpretation after BRAF immunostain. 
positive for BRAF staining, 13 (72\%) of these were histologically diagnosed as HPs, and five $(28 \%)$ of them diagnosed as $\mathrm{SSA} / \mathrm{Ps}$. Of the $16(47 \%)$ BRAF negative case in the study group, $12(75 \%)$ of these were histologic diagnosed as HPs, and four $(25 \%)$ of them diagnosed as SSA/Ps. $\beta$-catenin failed to show aberrant nuclear staining in any of the SSA/P or HPs in either the study or control group.

\section{Clinical follow-up}

Clinical follow-up information was available for 10 of the study cases $(36 \%$, mean follow-up time: 10 months, range: 3 to 75 months). One patient in the SSA/P group developed cancer 42 months after the diagnosis of SSA/P where it was completely resected with negative margin status. The tumor was located in the same region as the index polyp, which was the sigmoid colon. In addition, two of the SSA/P cases $(22 \%)$ exhibited adenoma-like low-grade dysplasia during follow-up, both in the ascending colon, with no carcinoma identified on follow-up. Of note, one other patient was diagnosed with cancer 1 month prior to the diagnosis of SSA/P. The tumor was initially found in the descending colon whereas the polyp was in the ascending colon. Only one case with HP developed HP in the same segment; no adenoma-like dysplasia or cancer was noted during follow-up in the HP patients in the study group.

\section{Discussion}

We studied the clinical, histological, and immunohistochemical features of SPs arising in the setting of IBD in our referral center cohort in a 10-year span (2004 - 2014). The caveat is that these lesions are rather uncommon, as a recent Mount Sinai Hospital study reported a prevalence of only $1.2 \%$ [13] in their surveillance patients, which is similar to the $2.1 \%$ in a recent Mayo Clinic study [20].

BRAF stain using the currently available mutant-specific antibody highlights a known mutation that has been associated with colorectal cancer [21]. The BRAF stain showed similar staining pattern in both the study group and control group, with approximately $50 \%$ positivity in both SSA/P and HPs. This finding is consistent with the previous molecular findings by Ko et al [13] which showed no difference in BRAF mutation status. Furthermore, they also looked into other mutations like $K R A S$ and showed similar findings. In follow-up of the SSA/P study group, one patient developed adenocarcinoma in the same location of the previous SSA/P 42 months later. But given the rare incidence of a single case, the clinical or scientific significance of this event cannot be characterized. On follow-up of the HP study group, only one patient developed recurrent HPs in the same location (sigmoid) 10 months later. The patient also had focal background active colitis and multiple hyperplastic BRAF positive polyps to begin with.

The SPs were also universally negative for $\beta$-catenin nuclear staining, which is consistent with the hypothesis that SPs evolve from a different pathway from traditional adenomatous polyps. One previous study showed that nuclear staining for $\beta$-catenin can be seen in $13.0 \%$ of normal colonic mucosa, and $60.5 \%$ and $79.2 \%$ for tubular adenoma with dysplasia and tubular adenoma with carcinomatous changes, respectively [18]. Here the SPs were all negative for $\beta$-catenin nuclear staining. A recent study conducted by Ko et al [13] also found that IBD patients with SPs with low grade dysplasia to have similar prognosis with IBD patients with non-SPs and low grade dysplasia. Their study also focused on the molecular basis of these SPs and found that the $B R A F$ and $K R A S$ mutation status was similar to those reported in sporadic SPs in non-IBD setting. Of note, they were able to get molecular testing in only approximately $50 \%$ of all their polyps.

Sporadic SPs, especially SSA/P, are known precursors for carcinoma [14, 22, 23]. However, studies on these lesions in IBD are limited. Several other studies in the past have focus on the topic of "serrated epithelial changes" and some categories of "SPs" [24-28] in the IBD setting which looks extensively into the correlation between dysplasia and malignancy. Of note, only one patient developed subsequent adenocarcinoma during follow-up and one patient had cancer prior to the diagnosis of SSA/P. Two additional patients in the SSA/P group developed adenoma-like dysplasia compared to none in the HP group. Other studies by Shen et al [14] and Jackson et al [15] found a slightly increased risk of metachronous dysplasia but this was not statistically significant. The presence of dysplasia in these polyps may also play an important risk factor for developing subsequent neoplasia as reported by Ko et al [13]. Despite some emerging evidence within literature and in our study, a recommendation for more aggressive surveillance or treatment cannot be made at the moment.

Overall, the histologic and immunohistochemical features were similar to non-IBD controls. Endoscopically, most of these lesions were detectable, identified as polyps ( $90 \%)$, and could be easily biopsied. Our findings are in keeping with the most recently published literature. Jackson et al [15] similarly reports that SPs in IBD show similar histology to their nonIBD counterparts; in addition, they found no correlation of occurrence of these lesions with the background inflammation. Only three of our cases had active background inflammation precluding further assessment.

We acknowledge several important limitations to this study. First, is the retrospective nature of the study, there is bias introduced with case selection. Second, the number of patients studied was relatively small due to the rarity of this entity. Therefore, the results of this study might be underpowered. Third, some of the clinical and endoscopic information was limited and not uniformly recorded. Lastly, although we gathered information on the staining results of immunohistochemical stains, these results are less specific compared to other more advanced molecular techniques to differentiation. The strengths of the study are well-controlled study group with detailed histologic and clinical descriptions.

We conclude that the clinical, pathologic, and immunophenotypic characteristics of SPs in patients with IBD resemble those of their sporadic counterparts. They share overlapping histologic and immunophenotypic features, as well as molecular characteristics [13]. Although the patients with SSA/P and IBD group had developed more "neoplasia" compared to the HP patients with IBD, which may infer an increased risk in 
this group, further conclusions and recommendations cannot be made at this point. Still, our study provides valuable information to the limited body of literature.

\section{Disclosures}

All authors have no financial disclosure.

\section{Reference}

1. Beaugerie L, Itzkowitz SH. Cancers complicating inflammatory bowel disease. N Engl J Med. 2015;372(15):14411452.

2. Sugita A, Sachar DB, Bodian C, Ribeiro MB, Aufses AH, Jr., Greenstein AJ. Colorectal cancer in ulcerative colitis. Influence of anatomical extent and age at onset on colitiscancer interval. Gut. 1991;32(2):167-169.

3. Jess T, Loftus EV, Jr., Velayos FS, Winther KV, Tremaine WJ, Zinsmeister AR, Scott Harmsen W, et al. Risk factors for colorectal neoplasia in inflammatory bowel disease: a nested case-control study from Copenhagen county, Denmark and Olmsted county, Minnesota. Am J Gastroenterol. 2007;102(4):829-836.

4. Gupta RB, Harpaz N, Itzkowitz S, Hossain S, Matula S, Kornbluth A, Bodian C, et al. Histologic inflammation is a risk factor for progression to colorectal neoplasia in ulcerative colitis: a cohort study. Gastroenterology. 2007;133(4):1099-1105; quiz 1340-1091.

5. Eaden JA, Mayberry JF, British Society for Gastroenterology, Association of Coloproctology for Great Britain and Ireland. Guidelines for screening and surveillance of asymptomatic colorectal cancer in patients with inflammatory bowel disease. Gut. 2002;51(Suppl 5):V10-12.

6. Cairns SR, Scholefield JH, Steele RJ, Dunlop MG, Thomas HJ, Evans GD, Eaden JA, et al. Guidelines for colorectal cancer screening and surveillance in moderate and high risk groups (update from 2002). Gut. 2010;59(5):666-689.

7. Laine L, Kaltenbach T, Barkun A, McQuaid KR, Subramanian V, Soetikno R, Panel SGD. SCENIC international consensus statement on surveillance and management of dysplasia in inflammatory bowel disease. Gastrointest Endosc. 2015;81(3):489-501 e426.

8. Gaidos JK, Bickston SJ. How to optimize colon cancer surveillance in inflammatory bowel disease patients. Inflamm Bowel Dis. 2016;22(5):1219-1230.

9. Riddell RH, Goldman H, Ransohoff DF, Appelman HD, Fenoglio CM, Haggitt RC, Ahren C, et al. Dysplasia in inflammatory bowel disease: standardized classification with provisional clinical applications. Hum Pathol. 1983;14(11):931-968.

10. Soetikno R, Kaltenbach T, McQuaid KR, Subramanian V, Kumar R, Barkun AN, Laine L. Paradigm shift in the surveillance and management of dysplasia in inflammatory bowel disease (West). Dig Endosc. 2016;28(3):266-273.

11. Snover DC. Update on the serrated pathway to colorectal carcinoma. Hum Pathol. 2011;42(1):1-10.
12. Sweetser S, Smyrk TC, Sinicrope FA. Serrated colon polyps as precursors to colorectal cancer. Clin Gastroenterol Hepatol. 2013;11(7):760-767; quiz e754-765.

13. Ko HM, Harpaz N, McBride RB, Cui M, Ye F, Zhang D, Ullman TA, et al. Serrated colorectal polyps in inflammatory bowel disease. Mod Pathol. 2015;28(12):1584-1593.

14. Shen J, Gibson JA, Schulte S, Khurana H, Farraye FA, Levine J, Burakoff R, et al. Clinical, pathologic, and outcome study of hyperplastic and sessile serrated polyps in inflammatory bowel disease. Hum Pathol. 2015;46(10):1548-1556.

15. Jackson WE, Achkar JP, Macaron C, Lee L, Liu X, Pai RK, Lopez R, et al. The Significance of Sessile Serrated Polyps in Inflammatory Bowel Disease. Inflamm Bowel Dis. 2016;22(9):2213-2220.

16. Noffsinger AE. Serrated polyps and colorectal cancer: new pathway to malignancy. Annu Rev Pathol. 2009; 4:343-364.

17. O'Brien MJ. Hyperplastic and serrated polyps of the colorectum. Gastroenterol Clin North Am. 2007;36(4):947968, viii.

18. Murakami T, Mitomi H, Saito T, Takahashi M, Sakamoto N, Fukui N, Yao T, et al. Distinct WNT/beta-catenin signaling activation in the serrated neoplasia pathway and the adenoma-carcinoma sequence of the colorectum. Mod Pathol. 2015;28(1):146-158.

19. Snover D, Ahnen DJ, Burt RW, Odze RD. Serrated polyps of the colon and rectum and serrated ("hyperplastic") polyposis. In: Bosman FT, Carneiro F, Hruban RH, Theise ND, editors. WHO classification of tumours of the digestive system, 4th ed. Lyon: IARC; 160-165.

20. Johnson DH, Khanna S, Smyrk TC, Loftus EV, Jr., Anderson KS, Mahoney DW, Ahlquist DA, et al. Detection rate and outcome of colonic serrated epithelial changes in patients with ulcerative colitis or Crohn's colitis. Aliment Pharmacol Ther. 2014;39(12):1408-1417.

21. Weisenberger DJ, Siegmund KD, Campan M, Young J, Long TI, Faasse MA, Kang GH, et al. CpG island methylator phenotype underlies sporadic microsatellite instability and is tightly associated with BRAF mutation in colorectal cancer. Nat Genet. 2006;38(7):787-793.

22. Lu FI, van Niekerk de W, Owen D, Tha SP, Turbin DA, Webber DL. Longitudinal outcome study of sessile serrated adenomas of the colorectum: an increased risk for subsequent right-sided colorectal carcinoma. Am J Surg Pathol. 2010;34(7):927-934.

23. Hiraoka S, Kato J, Fujiki S, Kaji E, Morikawa T, Murakami T, Nawa T, et al. The presence of large serrated polyps increases risk for colorectal cancer. Gastroenterology. 2010;139(5):1503-1510, e1501-1503.

24. Rubio CA, Befrits R, Jaramillo E, Nesi G, Amorosi A. Villous and serrated adenomatous growth bordering carcinomas in inflammatory bowel disease. Anticancer Res. 2000;20(6C):4761-4764.

25. Kilgore SP, Sigel JE, Goldblum JR. Hyperplastic-like mucosal change in Crohn's disease: an unusual form of dysplasia? Mod Pathol. 2000;13(7):797-801.

26. Srivastava A, Redston M, Farraye FA, Yantiss RK, Odze RD. Hyperplastic/serrated polyposis in inflammatory 
bowel disease: a case series of a previously undescribed entity. Am J Surg Pathol. 2008;32(2):296-303.

27. Parian AM, Koh JM, Badamas J, Giardiello FM, Montgomery EA, Lazarev M. Serrated epithelial changes are associated with colorectal dysplasia in inflammatory bowel disease. Gastroenterology, 2013, 144(5): S-11.

28. Odze RD, Brien T, Brown CA, Hartman CJ, Wellman A, Fogt F. Molecular alterations in chronic ulcerative colitisassociated and sporadic hyperplastic polyps: a comparative analysis. Am J Gastroenterol. 2002;97(5):1235-1242. 